\title{
Ladybirds (Coleoptera: Coccinellidae) of Iran: 45 New Records from 10 Provinces
}

\author{
R. ABDOLAHI ${ }^{1}$, O. NEDVĚD ${ }^{2}$ and J. NOZARI ${ }^{1}$ \\ ${ }^{1}$ Department of Plant Protection, College of Agriculture and Natural Resources, \\ University of Tehran, Karaj, P.O.Box 31587-77871, Iran \\ ${ }^{2}$ Faculty of Science, Institute of Entomology, Biology Center, \\ University of South Bohemia, Branišovská 31, 37005 České Budějovice, Czechia
}

(Received: 6 December 2016; accepted: 2 January 2017)

\begin{abstract}
Forty-five records of 28 species of ladybird beetles (belonging to 15 genera, 8 tribes and 6 subfamilies of Coccinellidae) as new for particular provinces in Iran are presented. The ten provinces with new records are Alborz, Golestan, Hamadan, Hormozgan, Kohgiluyeh and Boyer-Ahmad, Mazandaran, North Khorasan, Sistan and Baluchistan, Tehran, and Zanjan.
\end{abstract}

Keywords: Lady beetle, ladybug, distribution, fauna.

So far, 142 species of ladybird beetles (Coccinellidae) have been recorded from all around of Iran (Abdolahi et al., 2016). The highest number of records comes from provinces Fars, Lorestan and Kerman. The most often recorded species are Hippodamia variegata (Goeze), Coccinella septempunctata Linnaeus, Oenopia conglobata (Linnaeus), Chilocorus bipustulatus (Linnaeus) and Adalia bipunctata (Linnaeus).

Since new records of ladybird species are published each year from diverse provinces of Iran (Abdolahi et al., 2016) it is obvious that the knowledge about the coccinellid fauna of Iran is still far from complete.

In this paper, we contribute to the current knowledge of coccinellid fauna in several Iranian provinces by our own sampling.

\section{Materials and Methods}

Our samplings were carried out during April to August 2014 and 2015 by sweeping, in the following ten Iranian provinces: Alborz, Golestan, Hamadan, Hormozgan, Kohgiluyeh and Boyer-Ahmad, Mazandaran, North Khorasan, Sistan and Baluchistan, Tehran, and Zanjan. Most samples were collected in agriculture ecosystems, particularly fields with intercropping system, where gardens and farmland were mixed along with different plant species.

\footnotetext{
* Corresponding author; e-mail: Rahim.abdolahi@ut.ac.ir
} 
The beetles were killed mostly by ethyl acetate and sometimes by freezing. Larger species were mounted on pins (number 0-2) and small species were glued on card labels. Out of the many species collected, we are reporting here only species that were not known for the above-mentioned provinces. So, all the species mentioned here are new records for the associated provinces according to the checklist of the family from Iran by Abdolahi et al. (2016). If not stated otherwise, the voucher specimens are deposited in the private collection of the first author.

For identification of the collected material the keys of Kovár (1995), Raimundo and van Harten (2000), Nedvěd (2015), Iablokoff-Khnzorian (1971), Fürsch et al. (1967), Kreissl and Uygun (1980) and Gourreau (1974) were used.

The subfamilies, tribes, genera, species and the provinces are ordered alphabetically. Taxonomy follows the catalogue of the Palaearctic Coccinellidae (Kovár, 2007). Previously known distribution in individual provinces of Iran is given according to Abdolahi et al. (2016) without mentioning the primary literature source.

\section{Results}

We provide 45 new records of 28 ladybird species belonging to 15 genera, 8 tribes and 6 subfamilies, sampled from the ten investigated provinces.

\section{Subfamily Chilocorinae \\ Tribe Chilochorini \\ Genus Exochomus Leach, 1815 \\ Exochomus quadripustulatus (Linnaeus, 1758)}

Known distribution in Iran: Chaharmahal and Bakhtiari, East Azerbaijan, Fars, Ilam, Isfahan, Kerman, Khuzestan, Kohgiluyeh and Boyer-Ahmad, Lorestan, Mazandaran, North Khorasan, Qazvin, Qom, Razavi Khorasan, Tehran, West Azerbaijan, Yazd, Zanjan.

Material examined: Alborz province: Nazarabd, $1227 \mathrm{~m}$ a.s.l., 3557'28"N 50³8'14"E, 1 adult, 9 Aug 2015, sampled by Soudabe Amini.

Hamadan province: Abas Abad road, $1905 \mathrm{~m}$ a.s.1., 3446'56"N 48²9'02"E, 2 adults, 21 Jul 2014, sampled by Rahim Abdolahi.

Exochomus undulatus (J. Weise, 1878)

Known distribution in Iran: Chaharmahal and Bakhtiari, Fars, Isfahan, Kerman, Kermanshah, Kohgiluyeh and Boyer-Ahmad, Lorestan, Markazi, Razavi Khorasan, South Khorasan, Tehran.

Material examined: Hamadan province: Sangestan, $1894 \mathrm{~m}$ a.s.1., 3447'08"N $48^{\circ} 35^{\prime} 51^{\prime \prime E}, 2$ adults, 9 Jul 2014, sampled by Rahim Abdolahi.

Zanjan province: Traom road, $1638 \mathrm{~m}$ a.s.1., $36^{\circ} 38^{\prime} 34^{\prime \prime} \mathrm{N} 48^{\circ} 31^{\prime} 32$ "E, 1 adult, 5 Feb 2015, sampled by Rahim Abdolahi. 
Genus Parexochomus Barovskij, 1922

Parexochomus melanocephalus (Zoubkoff, 1833)

Known distribution in Iran: Chaharmahal and Bakhtiari, Kerman, Lorestan, Razavi Khorasan.

Material examined: Hamadan province: Ekbatan dam villages, $1874 \mathrm{~m}$ a.s.l., 3446'48"N 48³7'06"E, 2 adults, 22 Jul 2014, sampled by Rahim Abdolahi.

Parexochomus pubescens (Küster, 1848)

Known distribution in Iran: Fars, Isfahan, Kerman, Khuzestan, Lorestan, Mazandaran, Sistan and Baluchestan, South Khorasan, Tehran.

Material examined: Alborz province: Nazarabd, $1227 \mathrm{~m}$ a.s.1., 3557'28"N 50³8'14"E, 3 adults, 9 Aug 2015, sampled by Soudabe Amini.

Hamadan province: Ekbatan dam villages, $1874 \mathrm{~m}$ a.s.1., 34 46'48"N 48 $37^{\circ} 06^{\prime \prime} \mathrm{E}$, 3 adults, 22 Jul 2014, sampled by Rahim Abdolahi.

Subfamily Coccinellinae

Tribe Coccinellini

Genus Adalia Mulsant, 1846

Adalia decempunctata (Linnaeus, 1758)

Known distribution in Iran: Alborz, Chaharmahal and Bakhtiari, Gilan, Golestan, Hamadan, Ilam, Isfahan, Kerman, Lorestan, Markazi, North Khorasan, South Khorasan, Tehran.

Material examined: Hormozgan province: Bandar Abbas, $10 \mathrm{~m}$ a.s.1., 2541'47"N 5751'53"E, 1 adult, 22 Apr 2015, sampled by Rahim Abdolahi.

Genus Aphidecta J. Weise, 1893

Aphidecta obliterata (Linnaeus, 1758)

Known distribution in Iran: Lorestan.

Material examined: Golestan province, Gorgan, $146 \mathrm{~m}$ a.s.1., 3650'26"N 5426'32"E, 1 adult, 14 Jun 2015, sampled by Rahim Abdolahi.

Genus Coccinella Linnaeus, 1758

Coccinella undecimpunctata Linnaeus, 1758

Known distribution in Iran: Alborz, Ardebil, East Azerbaijan, Fars, Gilan, Golestan, Hamadan, Ilam, Isfahan, Kerman, Khuzestan, Kohgiluyeh and Boyer-Ahmad, Lorestan, Mazandaran, North Khorasan, Semnan, Sistan and Baluchestan, South Khorasan, Tehran, West Azerbaijan, Yazd.

Material examined: Hormozgan province: Bandar Abbas, $10 \mathrm{~m}$ a.s.1., 27013'19"N $56^{\circ} 22^{\prime} 28^{\prime \prime} \mathrm{E}, 4$ adults, 21 Apr 2015, sampled by Rahim Abdolahi, deposited in the private 
collection of the first author; Bandar Abbas, $10 \mathrm{~m}$ a.s.1., 2541'47"N 57'51'53"E, 3 adult, 22 Apr 2015, sampled by Rahim Abdolahi.

Zanjan province: Traom road, 1638 m a.s.1., 36³8'34"N 48³1'32"E, 2 adults, 5 Feb 2015, sampled by Rahim Abdolahi.

Genus Oenopia Mulsant, 1850

Oenopia oncina (Olivier, 1808)

Known distribution in Iran: Chaharmahal and Bakhtiari, Fars, Hamadan, Ilam, Isfahan, Kerman, Kermanshah, Khuzestan, Lorestan, Markazi, Mazandaran, Qom, Razavi Khorasan, Sistan and Baluchestan, South Khorasan, Tehran, West Azerbaijan, Yazd.

Material examined: Alborz, Nazarabd, 1227 m a.s.1., 3557'28"N 50³8'14"E, 1 adult, 9 Aug 2015, sampled by Soudabe Amini.

North Khorasan province: Darkesh, 1045 m a.s.1., 37²6' 14 "N 56²4'05"E, 1 adult, 11 Jun 2015, sampled by Rahim Abdolahi.

Genus Propylea Mulsant, 1846

Propylea quatuordecimpunctata (Linnaeus, 1758)

Known distribution in Iran: Alborz, Alborz, Ardebil, Chaharmahal and Bakhtiari, Fars, Gilan, Golestan, Hamadan, Hormozgan, Isfahan, Kerman, Khuzestan, Kohgiluyeh and Boyer-Ahmad, Lorestan, Mazandaran, North Khorasan, Qom, Razavi Khorasan, Semnan, Tehran.

Material examined: Zanjan province: Traom road, $1638 \mathrm{~m}$ a.s.1., 36³8'34"N 48³1'32"E, 2 adults, 5 Feb 2015, sampled by Rahim Abdolahi.

\section{Subfamily Epilachinae}

Tribe Epilachini

Genus Chnootriba Chevrolat, 1837

Chnootriba elaterii (P. Rossi, 1794)

Known distribution in Iran: Bushehr, Fars, Gilan, Golestan, Hormozgan, Ilam, Isfahan, Kermanshah, Khuzestan, Lorestan, North Khorasan, Semnan, Tehran.

Material examined: Mazandaran province: University of Sari, $-12 \mathrm{~m}$ a.s.l., 36³9'46"N 53 3'57"E, 3 adults, 1 larva, 15 Jun 2015, sampled by Rahim Abdolahi.

\section{Subfamily Microweiseinae}

Tribe Serangiini

Genus Serangium Blackburn, 1889

Serangium montazerii Fürsch, 1995

Known distribution in Iran: Gilan, Golestan, Zanjan.

Material examined: Mazandaran province: Marzanabad, $1300 \mathrm{~m}$ a.s.1., 36 $21^{\prime} 50 " \mathrm{~N}$ 51 16'46"E, 1 adult, 16 Jun 2015, sampled by Rahim Abdolahi. 


\section{Subfamily Scymninae}

Tribe Hyperaspidini

Genus Hyperaspis Chevrolat, 1837

Hyperaspis polita Weise, 1885

Known distribution in Iran: Fars, Golestan, Kerman, Kermanshah, Khuzestan, Razavi Khorasan, South Khorasan.

Material examined: Alborz province: Nazarabd, $1227 \mathrm{~m}$ a.s.1., 3557'28"N 50³8'14"E, 2 adults, 9 Aug 2015, sampled by Soudabe Amini.

Hormozgan province: Rodan Bika, 178 m a.s.1., 27²1'48"N 57¹0'27"E, 1 adult, 21 Apr 2015, sampled by Rahim Abdolahi.

Hyperaspis quadrimaculata (Redtenbacher, 1843)

Known distribution in Iran: Alborz, Isfahan, Lorestan, Mazandaran.

Material examined: Hamadan province: Razan-Avaj road, $1952 \mathrm{~m}$ a.s.l., $35^{\circ} 28^{\prime} 02^{\prime \prime} \mathrm{N} 49^{\circ} 02^{\prime} 36^{\prime \prime} \mathrm{E}, 8$ adult, 7 Jul 2014, sampled by Rahim Abdolahi; Nahavand, Gian, $1552 \mathrm{~m}$ a.s.1., $34^{\circ} 11^{\prime} 18^{\prime \prime} \mathrm{N} 48^{\circ} 14^{\prime} 32^{\prime \prime E}, 3$ adults, 29 Jul 2015, sampled by Rahim Abdolahi.

Zanjan province: Traom road, $1756 \mathrm{~m}$ a.s.1., 36³4'35"N 48 $42^{\prime} 48^{\prime \prime} \mathrm{E}, 1$ adult, 5 Feb 2015, sampled by Rahim Abdolahi.

\section{Tribe Platynaspidini}

Genus Platynaspis Redtenbacher, 1844

Platynaspis luteorubra (Goeze, 1777)

Known distribution in Iran: Ardebil, Fars, Lorestan, South Khorasan, Tehran.

Material examined: Hamadan province: Ekbatan dam villages, 1944 m a.s.l.,

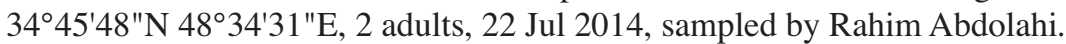

\section{Tribe Scymnini}

Genus Clitostethus J. Weise, 1885

Clitostethus arcuatus (Rossi, 1794)

Known distribution in Iran: Ardebil, Chaharmahal and Bakhtiari, Fars, Gilan, Golestan, Kerman, North Khorasan, South Khorasan.

Material examined: Tehran province: District 10, $1159 \mathrm{~m}$ a.s.1., $35^{\circ} 41^{\prime} 02^{\prime \prime} \mathrm{N}$ $51^{\circ} 21^{\prime} 45^{\prime \prime E}, 8$ adults, 20 Apr 2016, sampled by Maryam Safari, deposited in private collection of Maryam Safari. 
Genus Nephus Mulsant, 1846

Nephus bipunctatus (Kugelann, 1794)

Known distribution in Iran: Fars, Isfahan, Gilan, Kerman, Semnan, Zanjan.

Material examined: North Khorasan province: Bazkhaneh, 1213 m a.s.l., 37²4'5"N 57²0'18"E, 4 adults, 10 Jun 2015, sampled by Rahim Abdolahi.

Genus Scymnus Kugelann, 1794

Scymnus apetzi Mulsant, 1846

Known distribution in Iran: Chaharmahal and Bakhtiari, Fars, Gilan, Golestan, Hamadan, Isfahan, Khuzestan, Lorestan, Mazandaran, North Khorasan, Razavi Khorasan, South Khorasan, Tehran, West Azerbaijan.

Material examined: Alborz province: Nazarabd, $1227 \mathrm{~m}$ a.s.1., 35 57'28"N 50³8'14"E, 1 adult, 9 Aug 2015, sampled by Rahim Abdolahi.

Scymnus frontalis Fabricius, 1787

Known distribution in Iran: Gilan, Golestan, Isfahan, Kermanshah, Lorestan, Razavi Khorasan, Tehran, West Azerbaijan.

Material examined: Hamadan province, Ekbatan dam villages, $1875 \mathrm{~m}$ a.s.l., $34^{\circ} 46^{\prime} 48^{\prime \prime} \mathrm{N} 48^{\circ} 37^{\prime} 06^{\prime \prime} \mathrm{E}, 7$ adults, 22 Jul 2014, sampled by Rahim Abdolahi.

North Khorasan province: Darkesh, $1045 \mathrm{~m}$ a.s.1., 37²6'14"N 56 $45^{\prime} 05^{\prime \prime} \mathrm{E}, 10$ adults, 11 Jun 2015, sampled by Rahim Abdolahi.

Zanjan province: Traom road, $1638 \mathrm{~m}$ a.s.1., 36³8'34"N 48 $31^{\prime} 32^{\prime \prime E}, 5$ adults, 5 Feb 2015, sampled by Rahim Abdolahi.

Scymnus nigrinus Kugelann, 1794

Known distribution in Iran: Ardebil, Isfahan.

Material examined: Hormozgan province: Minab, $42 \mathrm{~m}$ a.s.1., 35 $53^{\prime} 23^{\prime \prime} \mathrm{N}$ 51ํㄴ'10"E, 1 adult, 22 Apr 2015, sampled by Rahim Abdolahi.

North Khorasan province: Esfarayen-Shirvan dirt road, 1249 m a.s.1., 37²14'36"N 5754'40"E, 1 adult, 11 Jun 2015, sampled by Rahim Abdolahi.

Scymnus nubilus (Mulsant, 1850)

Known distribution in Iran: Hormozgan, Lorestan.

Material examined: Kohgiluyeh and Boyer-Ahmad province: Yasuj, $1772 \mathrm{~m}$ a.s.1., 30³8'47"N 5135'56"E, 2 adults, 17 Apr 2015, sampled by Rahim Abdolahi.

Sistan and Baluchistan province: Zarabad-Chabahar road, $12 \mathrm{~m}$ a.s.1., 25 28'54"N 60³2'36"E, 10 adults, 22 Apr 2015, sampled by Rahim Abdolahi.

Zanjan province: Traom road, $1756 \mathrm{~m}$ a.s.1., 36 $34^{\prime} 35^{\prime \prime} \mathrm{N} 48^{\circ} 42^{\prime} 48^{\prime \prime} \mathrm{E}, 1$ adult, 5 Feb 2015, sampled by Rahim Abdolahi. 
Scymnus magnomaculatus Fürsch, 1958

Known distribution in Iran: Fars, Gilan, Golestan, Razavi Khorasan, Sistan and Baluchestan.

Material examined: Hamadan province: Ekbatan dam villages, $1875 \mathrm{~m}$ a.s.l., 34 $45^{\prime} 39^{\prime \prime} \mathrm{N} 48^{\circ} 34^{\prime} 55^{\prime \prime} \mathrm{E}, 2$ adults, 22 Jul 2014, sampled by Rahim Abdolahi; Malayer road, Siahkamar, 2024 m a.s.1., 34¹0'35"N 48³8'15"E, 1 adult, 12 Aug 2015, sampled by Rahim Abdolahi.

North Khorasan province: Darkesh, 1045 m a.s.1., 37²6'14"N 56²4'5"E, 1 adult, 11 Jun 2015, sampled by Rahim Abdolahi.

\section{Scymnus rubromaculatus Goeze, 1777}

Known distribution in Iran: Ardebil, Fars, Gilan, Isfahan, Kermanshah, Lorestan, Semnan.

Material examined: Golestan province, Gorgan, $147 \mathrm{~m}$ a.s.1., 3650'26"N 542''ㄹ"E, 1 adult, 14 Jun 2015, sampled by Rahim Abdolahi.

Hamadan province: Manuchehri, $1807 \mathrm{~m}$ a.s.1., 34048'44"N 48 $28^{\prime} 58^{\prime \prime} \mathrm{E}, 3$ adults, 1 Jul 2014, sampled by Rahim Abdolahi.

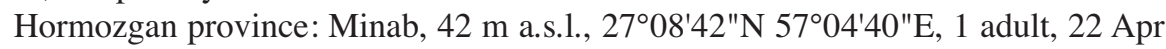
2015, sampled by Rahim Abdolahi.

North Khorasan province: Darkesh, 1045 m a.s.1., 37²2'14"N 5644'44"E, 1 adult, 11 Jun 2015, sampled by Rahim Abdolahi.

Zanjan province: Traom road, $1756 \mathrm{~m}$ a.s.l., 36³4'35"N $48^{\circ} 42^{\prime} 48^{\prime \prime} \mathrm{E}, 5$ adults, 5 Feb 2015, sampled by Rahim Abdolahi.

Mazandaran province: University of Sari, $-12 \mathrm{~m}$ a.s.1., 36 39'46"N 53 3'57"E, 3 adults, 15 Jun 2015, sampled by Rahim Abdolahi.

\section{Scymnus subvillosus Goeze, 1777}

Known distribution in Iran: Ardebil, Fars, Isfahan, Kerman, Lorestan, Markazi, Mazandaran, Razavi Khorasan, West Azerbaijan.

Material examined: Alborz province, Nazarabd, $1227 \mathrm{~m}$ a.s.l., 3557'28"N 50³8'14"E, 1 adult, 9 Aug 2015, sampled by Rahim Abdolahi.

Sistan and Baluchistan province: Zarabad-Chabahar road, $12 \mathrm{~m}$ a.s.1., 25 28 $54 " \mathrm{~N}$ 60³2'36"E', 2 adults, 22 Apr 2015, sampled by Rahim Abdolahi.

Scymnus suffrianioides Sahlberg, 1913

Known distribution in Iran: Isfahan, Kermanshah, Lorestan, Markazi, Razavi Khorasan, Tehran, Yazd.

Material examined: Hamadan province: Nahavand, Gian, 1552 m a.s.l., 34 $11^{\prime} 18^{\prime \prime} \mathrm{N} 48^{\circ} 14^{\prime} 32^{\prime \prime} \mathrm{E}, 4$ adults, 29 Jul 2015, sampled by Rahim Abdolahi. 
Scymnus syriacus (Marseul, 1898)

Known distribution in Iran: Ardebil, Chaharmahal and Bakhtiari, Fars, Gilan, Golestan, Hamadan, Isfahan, Kerman, Khuzestan, Lorestan, Markazi, North Khorasan, Razavi Khorasan, Semnan, South Khorasan, Zanjan, Tehran, Yazd.

Material examined: Sistan and Baluchistan province: Zarabad-Chabahar road, 12 m a.s.l., $25^{\circ} 28^{\prime} 54 " \mathrm{~N} 60^{\circ} 32^{\prime} 36^{\prime \prime E}, 1$ adult, 22 Apr 2015, sampled by Rahim Abdolahi; Zarabad, 15 m a.s.1., $25^{\circ} 39^{\prime} 04^{\prime \prime N} 59^{\circ} 20^{\prime} 15^{\prime \prime E}, 5$ adults, 22 Apr 2015, sampled by Rahim Abdolahi.

Scymnus testaceus Motschulsky, 1837

Known distribution in Iran: Lorestan.

Material examined: Hamadan province: Ekbatan dam villages, $1875 \mathrm{~m}$ a.s.l., 34ㄴ5'39"N 48³4'55"E, 1 adult, 22 Jul 2014, sampled by Rahim Abdolahi.

\section{Subfamily Sticholotidinae \\ Tribe Sticholotidini}

Genus Pharoscymnus Bedel, 1906

Pharoscymnus fleischeri (J. Weise, 1883)

Known distribution in Iran: Recorded from Iran by Kovář (2007) without mention of province.

Material examined: Hamadan province: Ekbatan dam villages, $1860 \mathrm{~m}$ a.s.l., 3447'9"N 48³7'7"E, 1 adult, 22 Jul 2014, sampled by Rahim Abdolahi.

Pharoscymnus flexibilis (Mulstant, 1853)

Known distribution in Iran: Fars.

Material examined: Sistan and Baluchistan province: Zarabad, $15 \mathrm{~m}$ a.s.l., $25^{\circ} 39^{\prime} 4$ "N 59 20'15"E, 5 adults, 22 Apr 2015, sampled by Rahim Abdolahi.

\section{Discussion}

The known distribution of 28 species of ladybirds in Iran was extended. In some common species, it was only a small addition to the knowledge already published, while Aphidecta obliterata, Scymnus testaceus, Pharoscymnus fleischeri and P. flexibilis were reported for the second time, and Scymnus nigrinus and S. nubilus were reported for the third time from the country. So far 15 species were reported from Hamadan province, but now the species number from Hamadan almost doubled (27 species).

Based on many recent additions to the fauna of Iran in the earlier published literature and in this paper, we expect many more records. Essentially, the less investigated provinces Kordestan and Qazvin should be visited for sampling ladybirds. 
Only after the knowledge of the distribution of more species within Iran will be more complete, analysis of their relations to larger biogeographical areas and division of the provinces of Iran to biogeographical groups will be possible.

\section{Acknowledgements}

We are grateful to Dr Mehdi Zare Khormizi (Department of Environment, Yazd, Iran) and Dr Ebrahim Sadeghi (Department of Forest Protection, Forest Research Institute of Iran, Tehran, Iran) for providing some literatures and identifying keys.

\section{Literature}

Abdolahi, R., Nozari, J., Allahyari, H. and Khormizi, M. (2016): Checklist and distribution of lady beetles (Coleoptera: Coccinellidae) in Iran. International J. of Animal Biosystematics 12, 1-35.

Fürsch, H., Capra, F. and Kreissl, E. (1967): Revision einiger europäischer Scymnus (s. str.)-Arten (Col., Coccinellidae). Mitteilungen der Abteilung für Zoologie und Botanik am Landesmuseum "Joanneum” in Graz. 28, 207-259.

Gourreau, J. M. (1974): Systematique de la tribu des Scymnini (Coccinellidae): contribution à l'étude de la faune de France. Annales de zoologie, ecologie animale (numero hors-serie): 1-221.

Iablokoff-Khnzorian, S. M. (1971): Synopsis des Hyperaspis palearctiques (Col. Coccinellidae). Annales de la Société Entomologique de France 7 163-200.

Kováŕ, I. (1995): Revision of the genera Brumus Muls. and Exochomus Redtb. (Coleoptera, Coccinellidae) of the Palaearctic Region. Part I. Acta Entomologica Musei Nationalis Pragae 44, 5-124

Kovár, I. (2007): Coccinellidae. In: I. Löbl and A. Smetana (eds): Catalogue of Palaearctic Coleoptera. Vol. 4. Stenstrup, Apollo Books, pp. 568-631.

Kreissl, E. V. and Uygun, N. (1980): Zur Kenntnis von Scymnus-Arten aus der Türkei. Mitteilungen der Abteilung für Zoologie und Botanik am Landesmuseum "Joanneum” in Graz 9, 189-202.

Nedvěd, O. (2015): Brouci čeledi slunéčkovití (Coccinellidae) střední Evropy. (Ladybird beetles (Coccinellidae) of Central Europe). Academia, Prague, 304 p.

Raimundo, A. A. and van Harten, A. (2000): An annotated checklist of the Coccinellidae (Insecta: Coleoptera) of Yemen. Fauna of Arabia 18, 211-244. 
